\title{
4
}

\section{Sketch-Based Interfaces for Parametric Modelling}

\author{
Ferran Naya, Manuel Contero \& Nuria Aleixos \\ Universidad Politécnica de Valencia \\ Spain \\ Joaquim A. Jorge \\ INESC-ID \& Universidade Técnica de Lisboa \\ Portugal \\ Pedro Company \\ Universidad Jaume I \\ Spain
}

\section{Introduction}

Sketching is still widely used by designers and engineers as it continues to be a useful and powerful tool that helps designers during the conception of a new product (Tversky, 2002). If engineers and designers generally use sketches the question is, why sketching is not integrated in the digital design process? Available Graphical User Interfaces (GUI) for CAD applications are still by and large constrained by the WIMP (Windows, Icons, Menus and Pointing) paradigm and current commercial CAD systems not support sketch-based design. Therefore, the problem is that the sketches continue to be unplugged to the rest of the design process. In other words, in spite of recent advances in Computer Aided Design, current $\mathrm{CAD}$ tools are not well suited to the initial design stages of product development, because many techniques and idioms characteristic of hand-made drawings cannot be used directly in CAD systems. To sum up, there is a disconnection between sketching and CAD tools in the new product development process and true Computer-aided Sketching (CASk) tools are required.

During last decades different research lines have been explored to improve the humancomputer interface in CAD systems. In this context, some CASk systems have been developed to support freehand drawings as a way to create and edit three-dimensional geometric models. These advanced CASk systems try to provide more functionality than paper or a whiteboard, giving an added value to sketching on a digital environment. This extra functionality usually has been directed either to improve the graphic quality of the sketch by means of a beautification process or it has been oriented to automatically transform the 2D sketch into a 3D model. Interest in CASk systems has increased in the last years as new hardware devices such as Tablet-PCs and LCD graphics tablets have been launched to the market. 
In this chapter we show our main contributions in the field of computer aided sketching. The aim of this work is to explore new interaction paradigms in CASk tools, geared at exploiting sketching skills of designers and engineers. Through this chapter the GEGROSS application developed by our research group (www.regeo.uji.es) will be used to illustrate the important concepts. GEGROSS is a CASk application than performs an online conversion of a raw sketch into a 3D model supporting parametric control of geometry.

\section{Sketch Based Interfaces and Modelling (SBIM)}

Over the last decades different research lines have been explored to improve the humancomputer interface in CAD systems. One of these new approaches is termed as "Sketchbased interfaces and modelling" (SBIM) that is an emerging research field oriented to the creation of new computer tools to promote a shift (Igarashi \& Zeleznik, 2007) to a new paradigm where sketches would be used as input to create 3D digital engineering models. Recent advances in SBIM applications promise better integration of sketching and CAD tools, integrating a paradigm shift to change the way geometric modelling applications are built, in order to focus on user-centric systems, rather than systems that are organized around the details of geometry representation. While most of the activity in this area in the past has been focused in off-line algorithms, where an application analyzes a complete sketch and then proposes a plausible 3D model, the growing focus on sketches and modelling has brought forth a new emphasis on approaches geared towards interactive applications. These interactive applications interpret in real time the input generated by a digitizing tablet and a pen, an approach also termed calligraphic interface (see Computers \& Graphics vol. 24, special issue "Calligraphic Interfaces: towards a new generation of interactive systems"). This kind of interface relies on the analysis of the pen strokes generated by the user, and exploits the space-time information provided by those to yield richer and more expressive interaction. A common feature of these systems is to use gestures (a special graphic symbol or stroke sequence) as commands (Fonseca and Jorge, 2001). These interfaces are specially suited to applications requiring capturing rough shapes and ideas, usually associated to the conceptual design stages of new product development. In these interfaces the artificial dialogue constraints imposed by the previous generation of WIMP user interfaces are removed and designers can interact with the computer in ways evocative of more traditional media, such as paper and pencil.

To sum up, there is a growing research interest in using freehand interaction and sketches as a way to create more natural interfaces, especially for the creation and edition of threedimensional geometric models. Digital sketching can offer an added value with respect to paper-and-pencil sketching, exploiting a more "natural" environment that does not disturb the user while he is creating the drawing. The availability of proper hardware as Tablet-PCs, electronic whiteboards and other devices supporting touch or stylus input is other of the reasons that support growing interest in this kind of interfaces.

The main requirement for designing an advanced CASk system should be to provide more functionality than paper or a whiteboard, trying to give an augmented digital paper. This extra capability with respect plain paper in some cases has been oriented to improve the graphic quality of the sketch by means of a beautification process as mentioned previously, or it has been oriented to automatically transform the 2D sketch into a 3D model. Here, it is possible to distinguish two principal approaches to transform the $2 \mathrm{D}$ sketch into a $3 \mathrm{D}$ 
model. One method relies on gesture alphabets as commands for generating objects from sketches (a gesture in this context represents a graphical symbol that is translated into a command). Examples of gestural systems are SKETCH (Zeleznik et al., 1996), Teddy (Igarashi et al., 1999), GIDeS (Pereira et al., 2000) and Blobmaker (De Araujo \& Jorge, 2003). Gestural systems provide predefined gesture alphabets that encode some geometric modelling operations; basically these systems substitute the selection of icons and menus by graphic gestures. The second approach, derived from computer vision, uses algorithms to reconstruct geometric objects from sketches that depict their two-dimensional projection. Examples of reconstruction systems are Stilton (Schweikardt \& Gross, 2000), Digital Clay (Turner et al., 2000) and CIGRO (Contero et al., 2005).

In summary, two basic alternatives exist to create 3D models from sketches: reconstruction based and gesture based. From these approaches the reconstruction based is the most transparent to users, since they have only to create a sketch which does not require a priori knowledge of a gestural command set. Chronologically, reconstruction systems appeared before gestural ones, because reconstruction systems took advantage of previous work in offline line drawing recognition. On the other hand, gestural systems require more elaborate recognition engines for distinguishing geometry information from gestural codes and most importantly, must provide elaborate user feedback in real time. Partly due to this and because of the restricted computing power available in earlier tablet PCs, some early systems avoided the disambiguation step by using icons and menus to explicitly provide this information to the system.

\section{The GEGROSS application}

The REGEO research group has developed in recent years the GEGROSS system, which is a CASk interactive application that converts raw sketches to three-dimensional models. The GEGROSS application follows the gestural approach and allows the user to generate threedimensional models using some gestural commands. In this system, it is possible to draw two-dimensional parametric freehand sections combined with the use of a simple gesture alphabet that encode some geometric modelling operations.

As can be seen in Fig. 1, in this application, the user introduces the freehand sketch directly onto a Tablet PC, using a reduced-instruction set calligraphic interface. The design goal of this interface is to create two-dimensional parametric sections and three-dimensional models in a very simple way, using the conventions of technical drawing to define the shape of the section. The user interface is designed to minimize the interaction with menus or icons in an attempt to emulate the traditional use of pen and paper.

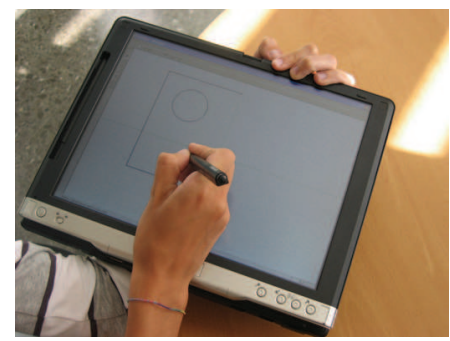

Fig. 1. User stroke input on a Tablet PC (GEGROSS) 
In the design of this system some assumptions have been taken to simplify the recognition process to interpret gestures. The main assumption is that the final user of this system will have a technical or engineering background. That means that users know the conventions of technical drawing, and the application is designed to recognize the typical drawing procedures that designers/engineers follow to create a technical sketch.

The gestural modelling process is organized in two stages. In first place a $2 \mathrm{D}$ profile is defined. To do this, the user introduces the geometry of the $2 \mathrm{D}$ section using a twodimensional parametric freehand sketch module called ParSketch (Naya et al., 2007). This module offers many of the features provided by current commercial parametric CAD applications as it is built on top of the most common parametric engine of the market (D-Cubed components from the SIEMENS firm, www.plm.automation.siemens.com). Later, in the second stage and also using gestures, it is possible to make an extrusion or a revolution of the parametric section generated in the previous stage to create a 3D solid model. Then this process, the user can continue sketching new 2D sections onto the faces of the generated object and applying the corresponding modelling gestures.

The ParSketch module implements a calligraphic interface to manage the geometric entities and the geometric constraints found in two-dimensional sections. The system distinguishes two modes of operation: one where the strokes made by the user are interpreted as geometric entities and other where the strokes are considered as commands. In table 1 the supported gestural alphabet is presented. Majority of gestures are inspired in the typical symbols used in technical drawing. When the user introduces a new stroke, ParSketch uses the drawing pressure as a mode discriminator (geometry or gesture). Then, the application interprets the type of stroke drawn by the user using a geometry recognizer (RecoGeo) or a gestural recognizer (RecoGes). Next, an automatic beautification stage is executed transforming the strokes in the corresponding geometry entities and constraint symbols.

The geometric recognizer RecoGeo supports complex strokes that after interpretation are split into its constituent primitives, allowing users to build simple sketches composed by line segments and arcs, which are automatically tidied and beautified. The application cleans up input data and adjusts edges to make sure they meet precisely at common endpoints in order to obtain geometrically consistent figures by filtering all defects and errors of the initial sketches that are inherent to their inaccurate and incomplete nature.

\begin{tabular}{|c|c|c|c|}
\hline $\begin{array}{c}\text { Constraint } \\
\text { gestures }\end{array}$ & Class & $\begin{array}{c}\text { Constraint } \\
\text { gestures }\end{array}$ & Class \\
\hline & Concentric & & Vertical \\
\hline & $\begin{array}{l}\text { Linea } \\
\text { dimension }\end{array}$ & H & Horizontal \\
\hline & $\begin{array}{l}\text { Diametral } \\
\text { dimension }\end{array}$ & 11 & Parallel \\
\hline & $\begin{array}{l}\text { Radial } \\
\text { dimension }\end{array}$ & 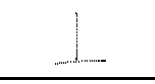 & Perpendicular \\
\hline & Tangent & 5 & $\begin{array}{l}\text { Cross-out } \\
\text { (erase) }\end{array}$ \\
\hline
\end{tabular}

Table 1. Gesture alphabet for constraining 2D geometry implemented in ParSketch 
Once the designer has introduced the complete outlined sketch, it can be edited, dimensioned and constrained using the gesture recognizer RecoGes. RecoGes has been developed to provide an alphabet of geometric/dimensional constraints to parameterise the sketches. In other words, if user wants to generate design alternatives, or adjusting some sketch to reach some dimensional condition, the system provides parametric capabilities and handwritten dimensional control to the two-dimensional freehand sections. Handwritten number recognition is provided by the Windows XP Tablet PC Edition operative system.

As explained before, the mode detection has been solved using the electronic pen pressure information, since the system is intended to be used by persons with basic engineering drawing skills. It can be said that line width is the mode-change feature when reading an engineering drawing. The usual practice is that thick lines are associated to geometry and thin lines to dimensions and other type of annotations. As line width is related to increasing pressure with the pencil while drawing, this information is used to discriminate among geometry or gesture. In other words, drawing making high pressure on the screen is intended for geometry input, while soft pressure is associated to auxiliary information. The user can configure a pressure level threshold to classify strokes as geometry or gestures.

An example of interaction with ParSketch is presented in Fig. 2. In this example the user draws the whole contour in 2.a. One single stroke is accepted as input, and it is later decomposed by the application into six rectilinear and connected strokes. When the application shows the beautified version (Fig. 2.b), the user adds another complex stroke composed by two segments and one arc. The geometry is then beautified (Fig. 2.d). In Fig. 2.e we can see the use of the scratching gesture to refine the geometry. Drawing this gesture is interpreted by the application as a command to delete those geometric entities intersecting the smallest quadrilateral that encloses the gesture. Then a parallel constraint is applied by simply sketching its associated gesture over the two segments we want to make parallel (see 2.f, 2.g, 2.h). Once the desired shape has been obtained, we can proceed with dimensional control. A first action is to draw a dimension without the dimension text (see Fig. 2.i). This is interpreted by the application as a measure command, and the current value of that dimension is shown, as seen in Fig. 2.j. If the user wants to change the current dimension value, he or she writes the new value next to the current one. Then the system regenerates and displays the new geometry (Fig. 2.k and 2.1). In this way, the system provides a very natural form of imposing the desired dimensions over the sketch.

As can be seen, once the designer has introduced the complete outlined sketch, it can be edited, dimensioned and constrained. In other words, the interface offers some innovative ways of controlling the shape after a beautified constrained model is presented to the user. The application manages two types of constraints and dimensions: "automatic" and "user defined". Automatic constraints and dimensions are those provided by the system. The "user defined" ones are sketched by user. As can be seen in Fig. 2, the user can add new constraints drawing their associated gestures (Table 1) near the geometric entities where they must be applied. These gestures can be written by the user to impose some desired constraint. In this context, the scratch gesture can be used to remove undesired constraints. The automatic beautification process (automatic constraints and dimensions) is in charge of adjusting the input sketch in real time and provides an immediate feedback to the user, because it operates as the user draws the sketch. 


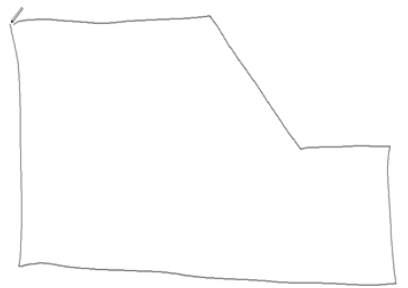

a)

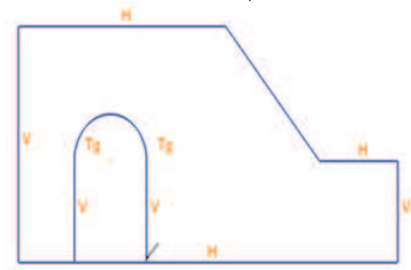

d)

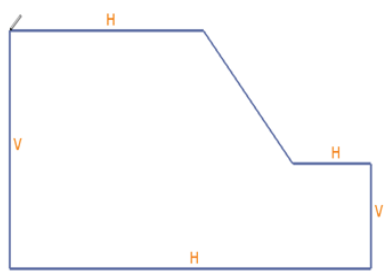

b)

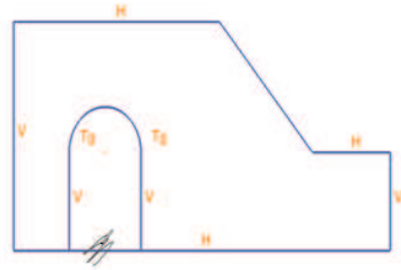

e)

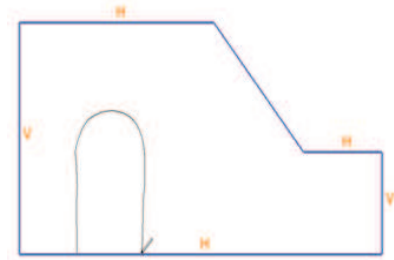

c)

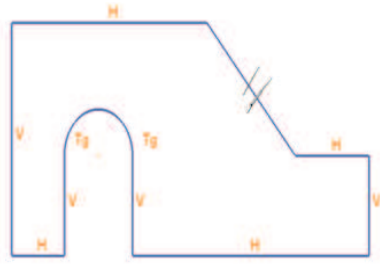

f)

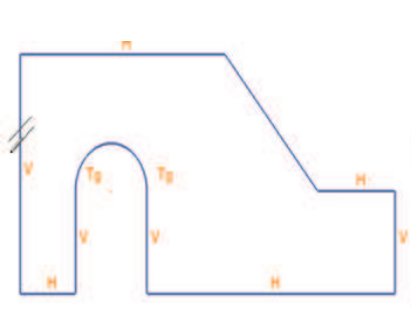

g)

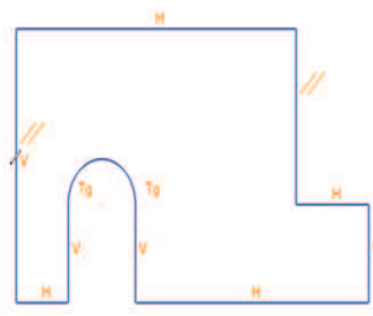

h)

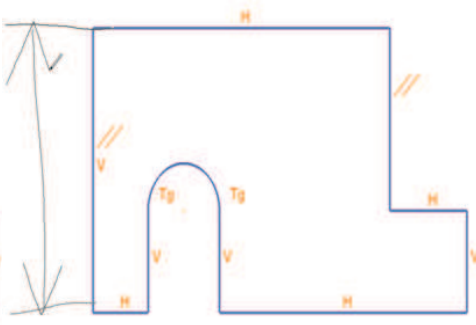

i)

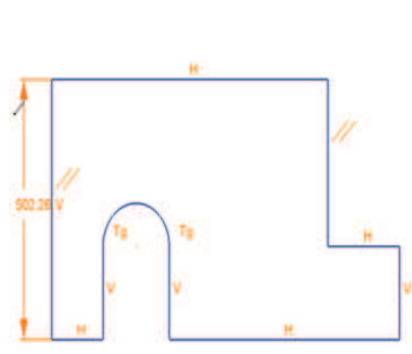

j)

Fig. 2. Sketching sequence in ParSketch

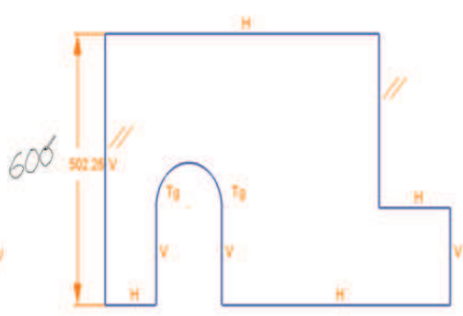

k)

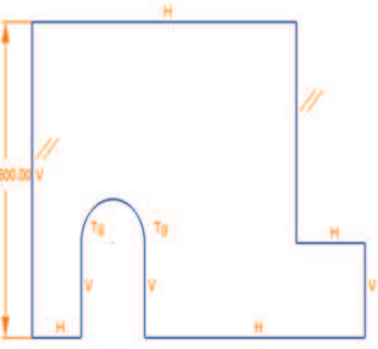

1)

The user can configure how the parametric engine controls the geometry. This control is implemented by a set of threshold values used to decide whether a geometric constraint is verified (see Fig. 3 for details) or not. The user has the possibility of enabling or disabling a specific constraint by an on/off selection box. Also it is possible to establish the order in which the constraints will be applied, using the "sequence" field in the dialog box presented in Fig. 3. These tolerance settings are intended to provide a tool for controlling the 
beautification action. Some of the supported constraints are: coincident (if a coincident constraint is defined between a point and any geometry then this implies that the point lies on the geometry), concentric, parallel, tangent, equal radius (it implies that the radii of the geometries are the same), perpendicular, equal distance (this constraint is used for search geometries with the same length), distance, angle and radius.

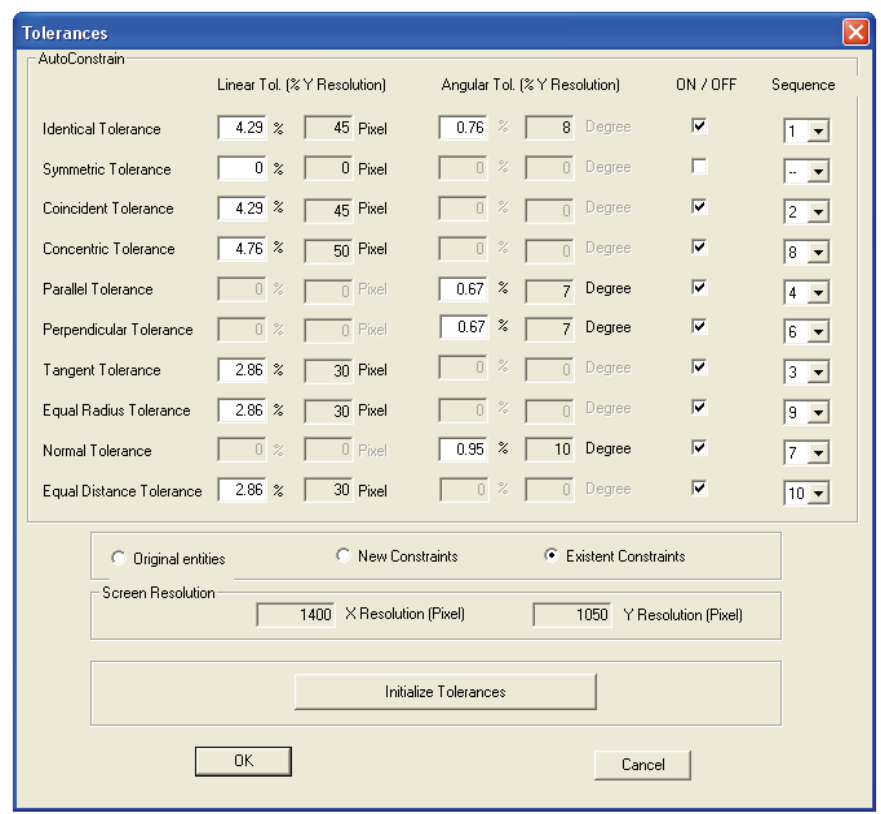

Fig. 3. Tolerance settings

Once a two dimensional section has been defined with the ParSketch module it is possible, using gestures, to make an extrusion or a revolution of the parametric section to create a 3D model. Then this process can continue sketching new 2D sections onto the faces of the generated object and applying the corresponding modelling gestures. In this second stage the command set includes the three gestures listed in Table 2.

\begin{tabular}{|l|l|}
\hline Modeling gestures & \multicolumn{1}{|c|}{ Class } \\
\hline & Extrusion \\
\hline & Revolve-right \\
\hline & Revolve-left \\
\hline
\end{tabular}

Table 2. Gesture alphabet for modelling operations implemented in GEGROSS 
The application recognizes the type of stroke drawn by the user using the gestural recognizer (RecoGes). Fig. 4, Fig. 5 and Fig. 6 show examples of modelling with the GEGROSS application.

The system uses the geometric kernel ACIS (www.spatial.com) to store the geometric entities. The points of the stroke are taken by means of the Wintab API (www.pointing.com), which is an open interface that directly collects pointing input from a digitizing tablet and passes it to applications. This API allows retrieving additional information as the pressure the user applies at each point of the stroke over the tablet.
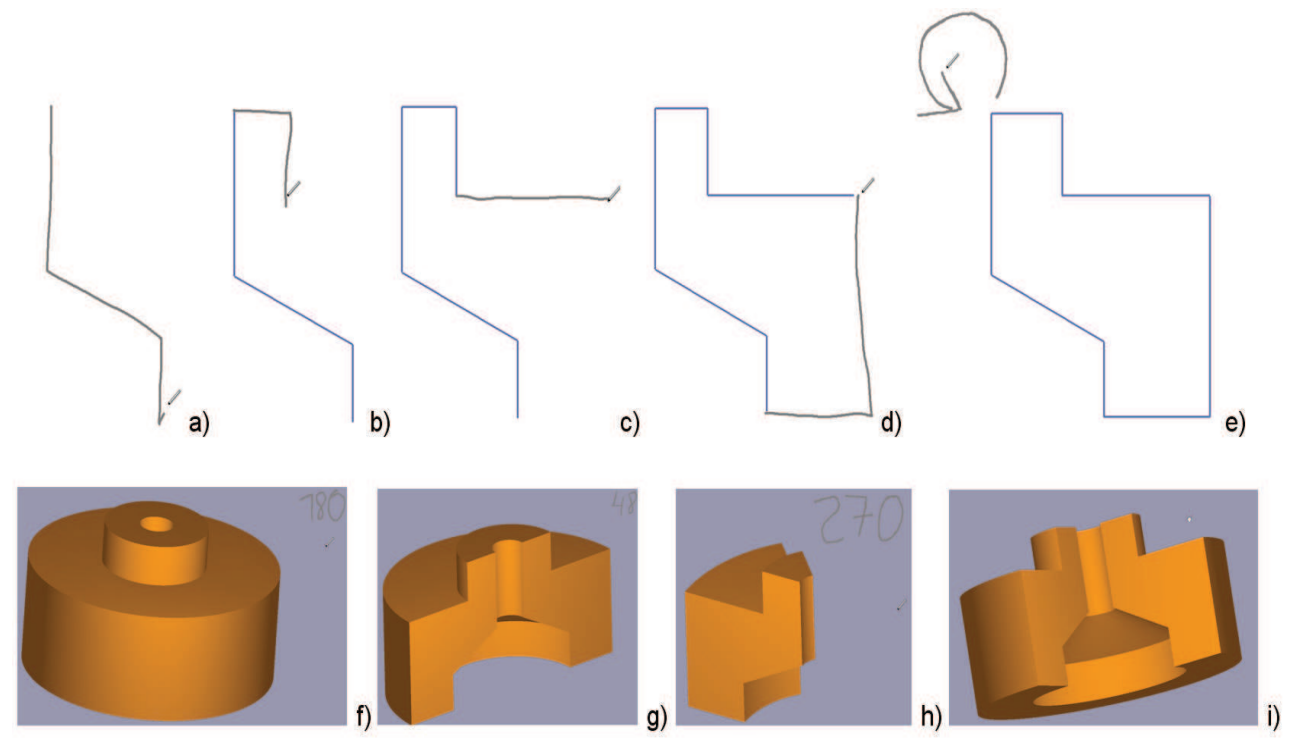

Fig. 4. Modelling sequence in GEGROSS. Example of revolution shape
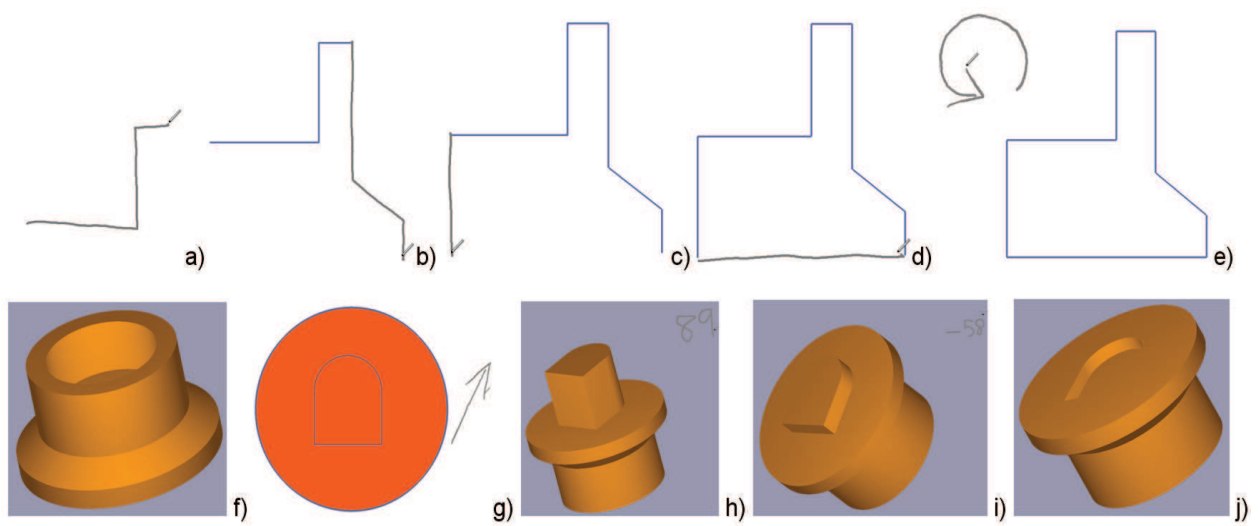

Fig. 5. Modelling sequence in GEGROSS. Example combining revolution and extrusion 


\section{Sketching-Based vs. WIMP Interfaces for Parametric Drawing}

From a theoretical point of view we can show that if the sketching application supports complex strokes, i.e. strokes composed by several basic primitives as line segments and arcs (see Fig. 7 as an example) this means a potential advantage over WIMP interaction. For instance, analyzing sections composed exclusively by arcs and line segments, we can make an approximated calculation of the number of interactions required by a WIMP application to complete the drawing task.

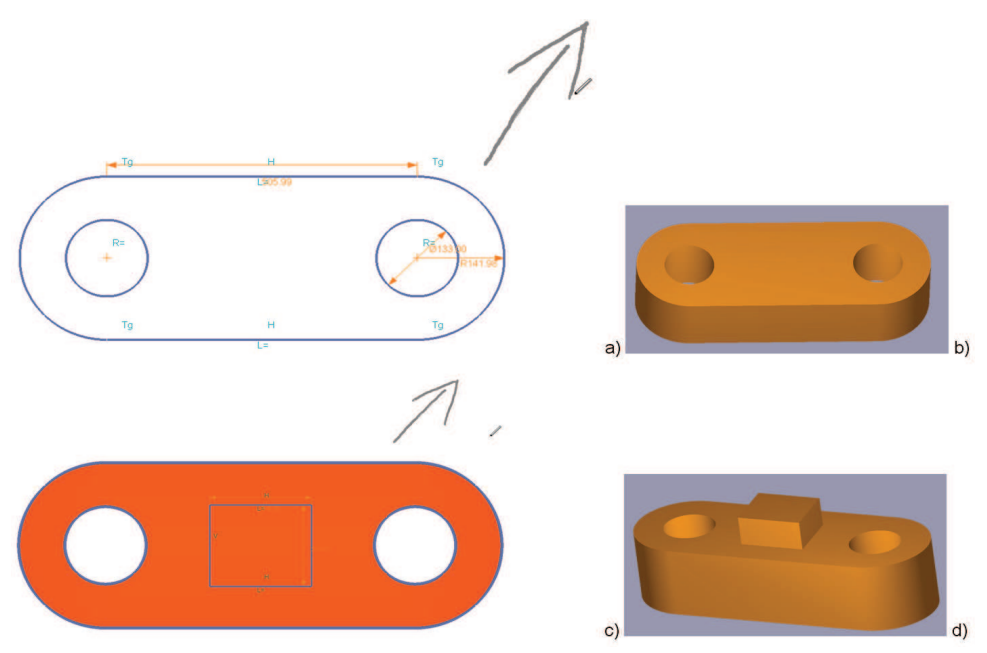

Fig. 6. Modelling sequence in GEGROSS. Example of extrusion-modelled shape

Usually one interaction is required to initiate the drawing process (one mouse click) and another one for finishing (a double click or pressing the enter key, for example). For drawing the line segments and tangent arcs in Fig. 7 two more interactions per elements are required: one is for defining the connecting vertex and the other for the selection of the proper geometric constraint as the horizontal, vertical, perpendicular or tangent conditions in this example. We count for this second interaction although, in modern parametric sketchers, geometric constraints are dynamically added as the user moves the drawing cursor. Only after the user detects the proper constraint is when he/she introduces the next entity vertex. This requires user attention, so we add it to the global number of interactions.

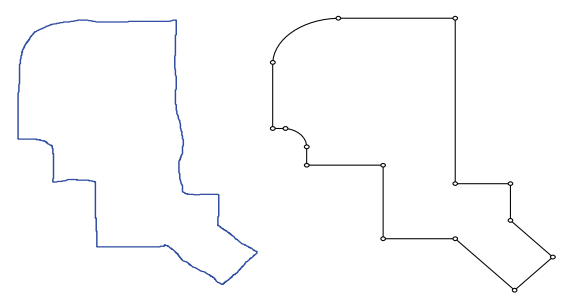

Fig. 7. Automatic segmentation vs. explicit drawing 
The last term in the calculation of total number of interactions is related to primitive switching operation. When the user wants to link for example a tangent arc to a previous polyline, he/she must spend one interaction, providing this information to the system (using for example a contextual menu or icon selection), and then spend a second interaction to come back to polyline mode.

In sum, if $n_{l}$ and $n_{a}$ represents the number of line segments and arcs respectively, the total number of interactions $N$ spent by the user is:

$$
N=2+2\left(n_{l}+n_{a}\right)+2 n_{a}
$$

Even for not too complex figures $(N=36$ in figure 4$)$ the last equation shows that although a user could employ several strokes to complete the shape and require some corrections to overcome recognition errors by the sketching application, there is a wide margin to compete with WIMP-based interaction in terms of efficiency. So it is feasible to implement a robust geometric segmentation and recognition to keep advantage over WIMP interaction. We think that this is one of the keys for success in providing a real alternative or at least a complement to a WIMP interface. But as Igarashi and Zeleznik noted (Igarashi \& Zeleznik, 2007), we must adapt the design of our applications to exploit the pen's intrinsic capacity for rapid, direct, modeless and expressive 2D input.

To improve segmentation results, our system can be adapted to each user way of sketching by means of a tolerance control panel previously described (see Fig. 3) that defines some key parameters for improving recognition. As explained before, the mode detection has been solved using the electronic pen pressure information, since GEGROSS is intended to be used by persons with basic engineering drawing skills.

In relation with other typical operations in a parametric 2D application, as imposing geometric constraints or performing dimensional control, the number of interactions required by both systems is similar. So we can conclude that from the efficiency point of view the sketch based approach is a viable option.

\subsection{Usability Study}

The usability of digital thinking sketches as opposed to traditional paper-and-pencil sketches was measured elsewhere (Company et al., 2006). In this analysis, we have centred our study in the user satisfaction component of usability (Hornbæk, 2006), following the usability definition provided by ISO 9241-11, where it stands for "extent to which a product can be used by specified users to achieve specified goals with effectiveness, efficiency and satisfaction in a specified context of use".

As noted previously, the main design goals of the GEGROSS application are:

- Expeditious creation of shapes composed by polylines, arcs, and circles.

- Dimensional and geometric shape-control though the use of technical drawing conventions.

The evaluation involved six CAD instructors and six students with parametric CAD experience. All but one of the CAD instructors were male with an average age of 55. All students were male with an average age of 24 .

We allowed 30 minutes for the evaluation, which had four parts: an overview of the system where some short videos showed the system operation, an instruction stage with a modified 
version of GEGROSS that explicitly informs the user about the recognized entities or gestures (typically 10 minutes were employed in this training), a drawing task, and a final discussion with participants. After the discussion, users filled a questionnaire to evaluate GEGROSS and express their comments about it.

Each participant used a Toshiba Tecra M4. This Tablet PC has a 14,1" screen, with a resolution of 1400x1050 dots, and employs Ms Window XP Tablet-PC Edition.

We asked users to accomplish three drawing tasks using the ParSketch module. Shapes presented in Fig. 8 where used to propose several drawing exercises. The first exercise was to create a parametric section similar to the left shape of Fig. 8. The other two exercises employed the other shapes, and the users had to create the shape and impose some dimensional and geometric constraints.
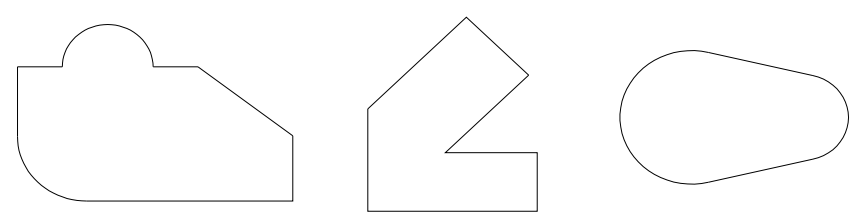

Fig. 8. Shapes for usability study

In relation with the evaluation of effectiveness, we have measured the accuracy and completeness with which users achieved the goals presented previously, using experts' assessment of the produced sketches. This assessment is based on the accuracy of the generated shapes. All the participants completed the requested drawing tasks satisfying all the conditions imposed to the generated shapes.

Efficiency has been measured taking into account the resources expended in relation to the accuracy and completeness with which users completed the drawing tasks. In our study we have used the task completion time and the number of events logged by a modified macro recording application. The most interesting result in this measure was the comparison between the best results obtained with the ParSketch module and the minimum number of interactions required by PTC's Pro/Engineer Wildfire 3 to complete the drawing tasks (this data are presented in Table 3). For all the participants in the study, this was their first contact with a Tablet-PC, and some of them had problems to control the pressure threshold that changes input mode.

\begin{tabular}{|l|c|c|}
\hline \multicolumn{1}{|c|}{ Exercise } & ParSketch (\# of strokes) & Pro/E (\# mouse click + \# menu selection) \\
\hline$\# 1$ (left) & 3 & $12+4$ \\
\hline \#2 (middle) & 1 & $8+1$ \\
\hline \#3 (right) & 4 & $10+4$ \\
\hline
\end{tabular}

Table 3. Efficiency comparison

From Table 3 we can extract a first topic of discussion. Is it comparable the mental effort to generate a stroke on the Tablet-PC with the equivalent mouse operations to define the same geometry? We think that for users with previous experience in sketching on plain paper, drawing is practically an automatic task, which requires less concentration and effort than 
the mouse operation. Perhaps this justifies that $100 \%$ of participants evaluated as easier, the use of the ParSketch module with respect to the CAD tools known by them.

Finally, user satisfaction has been measured using an adapted version of the QUIS Questionnaire (Chin,1998) using a 10 point scale from 0 to 9 . A selection of the questions is presented in Table 4. In general, all participants expressed a very positive attitude towards the application, and all of them learnt in a few minutes to use it. Majority of comments about the system came from the pressure-based mode selection and about recognition errors. With respect to the pressure, none of participants had had previous experience with pressure sensible application and this had a distracting effect, requiring some concentration effort to change from the geometry input mode to the gesture one. We think that with more time of use, this mode change would not require so much effort. Also we are thinking about the convenience of providing some kind of online indicator (feedback) of what kind of input is receiving the system. Now, the application uses a paradigm similar to drawing on plain paper. Thickness of the rendered stroke in screen is related to the pressure done by the user while it is drawing. We are thinking on a color-based indication system that will represent geometry strokes in one color, and gesture strokes in another different one. This color assignment should be done dynamically, because in this way, if the user inadvertently begins to draw the stroke in the wrong mode he/she can correct it on the fly.

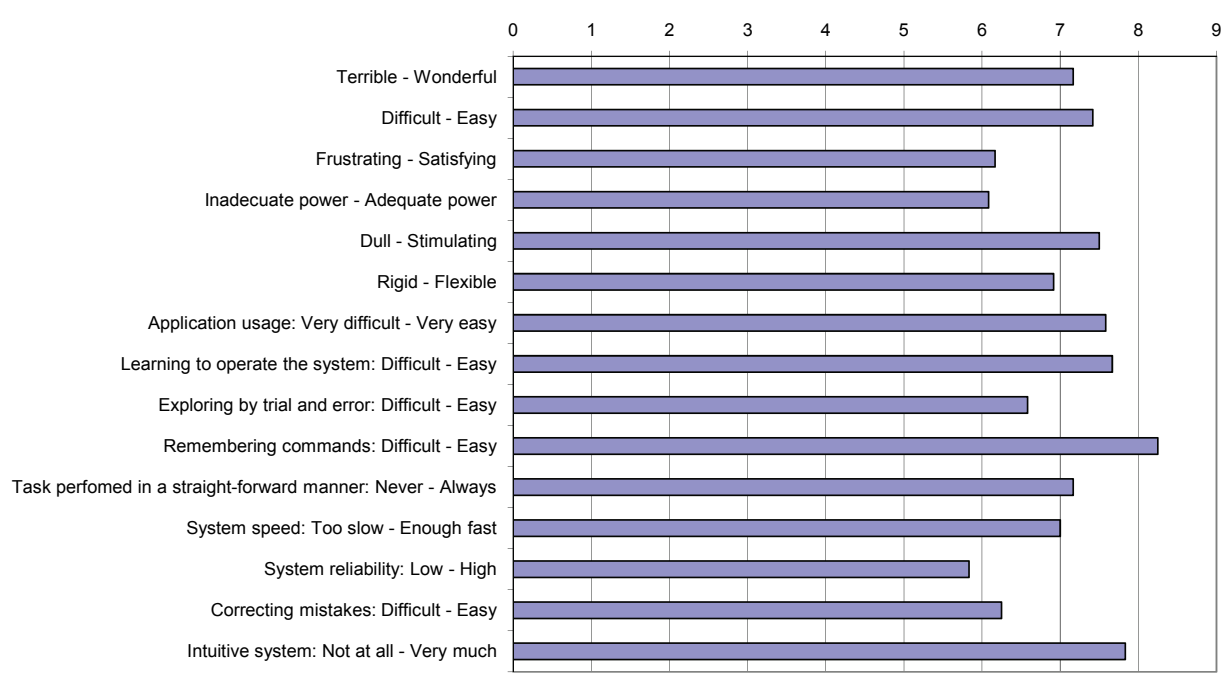

Table 4. User satisfaction measures

The other part of the comments about the system came from mistakes in the recognition process. This creates some kind of frustration in the user, when he/she draws a stroke or a gesture and a wrong interpretation is provided. The recognition rate for gesture recognition was 90 percent. Rates for geometry recognition were very variable, depending on the complexity of the generated stroke and the ability of the user creating the sketch.

In order to improve recognition results, we are studying the creation of a training mode in the application to adapt and tune recognition process to each user procedure of sketching. 


\section{Conclusion}

In this chapter, an approach to create three-dimensional parametric objects using a gesture alphabet has been described. The main objective of this work has been to provide dimensional and geometrical control over the sections in an easy and natural way. The system offers a very simple interface to create parametric sections with an interesting possibility: "dimensional control". In this way, user can impose some dimensional condition drawing the corresponding dimension and writing its value. This handwritten dimensions offer a natural and simple method to change dimension values that is known by any engineer.

Comparing the operation of the GEGROSS system with a standard WIMP parametric CAD application we can say that the basic functionality is practically equivalent. As can be seen in previous examples, usability is enhanced as well since the interface has been particularly tailored to detect standardized symbols. New symbols are not invented for any existing ones, but they are "borrowed" from the set of meaningful engineering symbols currently defined in the standards (ISO, ASME...). Therefore, the improvement in usability results from the fact that those symbols are commonplace for potential users: no learning is required, and unconscious user actions are readily interpreted by the computer.

In other words, learnability of GEGROSS has proven to be very high. Actually, users only have required ten minutes of introduction and demonstration before using the system. This in part is justified by the engineering background of participants. But GEGROSS has been specifically designed for this kind of users, trying to exploit their knowledge of technical drawing conventions and their sketching abilities. Perhaps this is one of the reasons of this positive reaction. Users feel that this tool adapts to them, not requiring a special effort for learning to use.

Preliminary tests have shown encouraging results and have concluded the GEGROSS application is a feasible alternative to current approach used in commercial CAD applications in order to create shapes of small or medium complexity. In that situation it presents a more effective modelling time, and it has been rated as easier to learn than comparable commercial applications. Users that have an engineering background find very natural the system behaviour, and the learning process to manage the application is very fast. Therefore, user satisfaction has been very high during the usability study. Users enjoy the simplicity of the system and its powerful control of geometry. However, improvements are needed to give a clearer feedback of pressure mode selection.

GEGROSS offers in many cases higher efficiency than a comparable WIMP application. This is much related to supporting complex strokes, i.e. strokes composed by mixed basic primitives as line segments and arcs, for defining the shape's geometry. However a high efficiency in terms of complex stroke support can have an undesired side effect: worse system effectiveness because of the increasing difficulty of the recognition and segmentation tasks. So we can conclude, than the best alternative for getting the best results is the combination of several medium complex strokes, instead of trying to define the whole geometry in one only stroke. Besides, the user can take advantage of the edition strokes (erase and the like) to follow another good strategy: recursive refinement of a first rough version of the stroke. It has some advantages. First, reduces the fail rate of the system. Second, forces the user to sketch in a more convenient way: concentrating in the major shapes, and letting the details for subsequent refinements. 
Acknowledgments. The Spanish Ministry of Science and Education and the European Union (Project DPI2007-01373) supported this work. It was also partially supported by the Portuguese Foundation of Science and Technology, under grant DecorAR POSC/EIA/59938/2004.

\section{References}

Chin, J.P.; Diehl, V.A. \& Norman, K.L. (1988). Development of an instrument for measuring user satisfaction of the human-computer interface. Proceedings of ACM Conference on Human Factors in Computing Systems, pp. 213-218, ISBN 0-201-14237-6, April 1998, ACM Press, New York, NY

Company, P.; Contero, M.; Naya F. \& Aleixos, N. (2006). A Study of Usability of Sketching Tools Aimed at Supporting Prescriptive Sketches, Proceedings of the 4th Eurographics Workshop on Sketch-Based Interfaces and Modeling, pp. 139-146, ISBN 3-905673-39-8, September 2006, Eurographics, Vienna

Contero, M.; Naya, F.; Company, P.; Saorín, J.L. \& Conesa J. (2005). Improving Visualization Skills in Engineering Education. IEEE Computer Graphics and Applications, Vol. 25, No. 5, (September/October 2005) 24-31, ISSN 0272-1716

De Araujo, B. \& Jorge, J. (2003). BlobMaker: Free-form modelling with variational implicit surfaces, Proceedings of $12^{\circ}$ Encontro Português de Computação Gráfica, pp. 17-26, October 2003, Porto

Fonseca, M. \& Jorge, J. (2001). Experimental Evaluation of an On-Line Scribble Recognizer. Pattern Recognition Letters, Vol. 22, No. 12 (October 2001), 1311-1319, ISSN 0167-8655

Igarashi, T.; Matsuoka, S. \& Tanaka, H. (1999). Teddy: a sketching interface for 3D freeform design, Proceedings of ACM SIGGRAPH '99, pp. 409-416, ISBN 0-20148-560-5, August 1999, publisher, Los Angeles, California

Igarashi, T. \& Zeleznik, B. (2007). Sketch based interaction. IEEE Computer Graphics and Applications, Vol. 27, No. 1, (January/February 2007) 26-27, ISSN 0272-1716

Hornbæk, K. (2006). Current Practice in Measuring Usability: Challenges to Usability Studies and Research. International Journal of Human-Computer Studies, Vol. 64, No. 2, (February 2006) 79-102, ISSN 1071-5819

Naya, F.; Contero, M.; Aleixos, N. \& Company, P. (2007). ParSketch: A Sketch-Based Interface for a 2D Parametric Geometry Editor. Lecture Notes in Computer Science, Vol. 4551, (August 2007) 115-124, ISSN 0302-9743

Pereira, J.; Jorge, J.; Branco, V. \& Nunes, F. (2000). Towards calligraphic interfaces: sketching 3D scenes with gestures and context icons, Proceedings of WSCG'2000 Conference, pp. ,ISBN 80-7082-612-6, February 2000, Skala V. Ed., Plzen

Schweikardt, E. \& Gross, M.D. (2000). Digital Clay: deriving digital models from freehand sketches. Autom. in Construction, Vol. 9, No. 1(Jan. 2000) 107-115, ISSN 0926-5805

Turner, A.; Chapmann, D. \& Penn, A. (2000). Sketching space. Computers \& Graphics, Vol. 24, No. 6, ( December 2000) 869-879, ISSN 0097-8493

Tversky, B. (2002). What Do Sketches Say About Thinking?. AAAI Spring Symposium Series - Sketch Understanding, pp. 148-152, ISBN 978-1-57735-153-5

Zeleznik, R.C.; Herndon, K.P. \& Hughes, J.F. (1996). SKETCH: an interface for sketching 3D scenes, Proceedings of SIGGRAPH '96, pp. 163-170, ISBN 0-89791-746-4, August 1996, ACM, New Orleans 


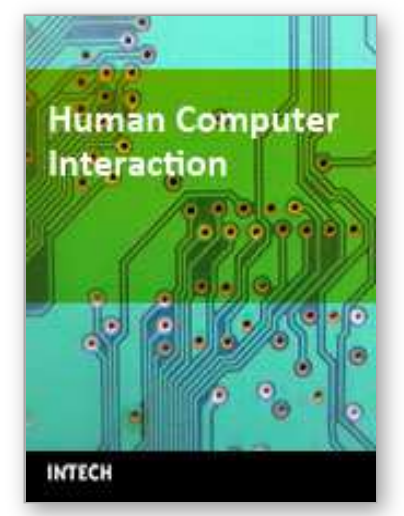

\section{Human Computer Interaction \\ Edited by loannis Pavlidis}

ISBN 978-953-7619-19-0

Hard cover, 522 pages

Publisher InTech

Published online 01, October, 2008

Published in print edition October, 2008

This book includes 23 chapters introducing basic research, advanced developments and applications. The book covers topics such us modeling and practical realization of robotic control for different applications, researching of the problems of stability and robustness, automation in algorithm and program developments with application in speech signal processing and linguistic research, system's applied control, computations, and control theory application in mechanics and electronics.

\section{How to reference}

In order to correctly reference this scholarly work, feel free to copy and paste the following:

Ferran Naya, Manuel Contero, Nuria Aleixos, Joaquim A. Jorge and Pedro Company (2008). Sketch-Based Interfaces for Parametric Modelling, Human Computer Interaction, Ioannis Pavlidis (Ed.), ISBN: 978-953-761919-0, InTech, Available from: http://www.intechopen.com/books/human_computer_interaction/sketchbased_interfaces_for_parametric_modelling

\section{INTECH}

open science | open minds

\section{InTech Europe}

University Campus STeP Ri

Slavka Krautzeka 83/A

51000 Rijeka, Croatia

Phone: +385 (51) 770447

Fax: +385 (51) 686166

www.intechopen.com

\section{InTech China}

Unit 405, Office Block, Hotel Equatorial Shanghai

No.65, Yan An Road (West), Shanghai, 200040, China

中国上海市延安西路65号上海国际贵都大饭店办公楼 405 单元

Phone: +86-21-62489820

Fax: $+86-21-62489821$ 
(C) 2008 The Author(s). Licensee IntechOpen. This chapter is distributed under the terms of the Creative Commons Attribution-NonCommercialShareAlike-3.0 License, which permits use, distribution and reproduction for non-commercial purposes, provided the original is properly cited and derivative works building on this content are distributed under the same license. 